\title{
Enhancing Electrochemical Detection by Scaling Solid State
}

\section{Nanogaps}

\author{
Gregory S. McCarty, Benjamin Moody, and Matthew K. Zachek \\ Joint Department of Biomedical Engineering, University of North Carolina at Chapel Hill and North \\ Carolina State University, Raleigh NC 27695
}

Gregory S. McCarty: gmccarty@unc.edu

\begin{abstract}
The ability to quickly and inexpensively fabricate planar solid state nanogaps has enabled research to be effectively performed on devices down to just a few nanometers. Here, nanofabricated electrode pairs with electrode-to-electrode spacings of $<4,6$ and $20 \mathrm{~nm}$ are utilized for monitoring an electroactive molecules, dopamine, in ionic solution. The results show a several order of magnitude enhancement of the electrochemical signal, collected current, for the solid state nanogaps with $6 \mathrm{~nm}$ electrode-electrode spacings as compared to traditional microelectrodes. The data from the $<4 \mathrm{~nm}$ and $20 \mathrm{~nm}$ solid state nanogaps verify that this enhancement is due to cycling of the redox molecules in the confined geometry of the nanogap. In addition the data collected for the $<4 \mathrm{~nm}$ nanogap emphasizes and reinforces that scaling does have limits and that as device sizes move to the few nanometer scale, the influence of a molecule's size and other physical properties becomes increasingly important and can eventually dominate the generated signals.
\end{abstract}

\begin{abstract}
Nanoscience is a maturing field that has the potential of offering unique solutions to a variety of societal problems. Many novel nanoscale techniques are being explored to satisfy unmet needs in a number of areas including chemical and biological monitoring. For example, there is much interest in developing of inexpensive chemical and biological detection schemes capable of monitoring small numbers (a few to a few thousands) of molecules in a straight forward manner, without labeling, in small (sub-picoliter) volumes of solution, as these types of advances are expected to have profound impact on areas from biodefense through medicine. Accordingly, there is much interest and research being performed in the nanosciences for creating ultra-sensitive monitoring schemes to meet these needs.

In an influential publication by Bard and co-workers, single molecule electrochemical detection was demonstrated by trapping a redox active molecule between a conductive surface and a conductive probe spaced nanometers apart.(1,2) The redox molecule cycled between the electrodes, undergoing repeated oxidations and reductions. This cycling led to measurable signals for even a single molecule.(2) While these works suggest exciting possibilities for the detection of small numbers of electroactive species in spatially constrained environments, the need to manipulate at least one of the probes in the system makes this method difficult to implement for commercial monitoring or screening applications. More recent studies have
\end{abstract}

(C) 2010 Elsevier B.V. All rights reserved.

Correspondence to: Gregory S. McCarty, gmccarty@unc.edu.

Publisher's Disclaimer: This is a PDF file of an unedited manuscript that has been accepted for publication. As a service to our customers we are providing this early version of the manuscript. The manuscript will undergo copyediting, typesetting, and review of the resulting proof before it is published in its final citable form. Please note that during the production process errors may be discovered which could affect the content, and all legal disclaimers that apply to the journal pertain. 
transitioned this concept to solid state devices. Sensitive and selective electrochemical monitoring based on redox species cycling has been demonstrated using interdigitated microelectrode arrays(3-5) and electrodes created inside nanofluidic systems.(6) In a recent publication, nanofluidic systems with electrodes separated by $55 \mathrm{~nm}$ were fabricated and utilized for electrochemical monitoring of cycling catecholamines.(6) This publication demonstrates that cycling of redox molecules can occur between electrodes spaced by nanometer distances and further highlights methodologies to utilize this process for sensitive, selective electrochemical monitoring. However, these studies do little to explore the relationship and limits between redox molecule cycling and the design of the confined nanoscale geometries in which this cycling occurs.

In this report, planar electrode pairs fabricated to have defined electrode-electrode spacings of just a few nanometers are utilized for electrochemical monitoring of a small number of molecules. These electrode pairs, often referred to as "solid state nanogaps" were fabricated using a combination of conventional and molecular lithographies and tested as reported in previous research.(7-9) These previous reports demonstrate how the exchange, addition or removal of molecular species can be probed using solid state nanogaps, but only after the samples were removed from solution and dried.(7-9) Here, the use of planar solid state nanogaps for the detection of electroactive molecular species in ionic solutions is described. Also, comparing three different electrode-electrode spacings showed that a significant "electrochemical enhancement" (an increase in the collected current/(electrode area*electroactive species concentration)) is achieved by nanogaps with electrode-electrode spacings of $6 \mathrm{~nm}$ due to the electroactive molecules cycling within nanogaps. A schematic representation of cycling is described in Figure 1, in this case dopamine cycles between dopamine (reduced species) and dopamine ortho-quinone (oxidized species). This cycling enables the detection of very few electroactive molecules. Furthermore the limit of scaling for cycling based electrochemical monitoring, at least for these devices, is demonstrated, as the charge transfer properties of the $<4 \mathrm{~nm}$ solid state nanogap differ significantly from those of the $6 \mathrm{~nm}$ structure in ionic solution. This technique, while still immature, offers significant possibilities in the detection of small numbers of molecules in extremely small volumes of solution and holds great potential for future detection applications.

The planar solid state nanogaps are fabricated using techniques that have been previously described.(7-11) Briefly, a clean silicon sample is oxidized to create an insulating substrate and an initial metallic film of $\mathrm{Cr}$ and Au with a final thickness of $\sim 110 \mathrm{~nm}$ is evaporated onto the substrate. Then photolithography and reactive ion etching are used to form the first electrodes of each of the electrode pairs, labeled Au\#1 in Figure 2. These first electrodes will be referred to as the initial or excitation electrode in the electrode pair throughout this manuscript. Next a molecular resist, typically composed of a single or multilayered molecular film, is grown selectively on the metallic features. The growth and utilization of this multilayer resist has been discussed in several publications.(7,8,10-13) Next, a second photolithography step is used to create the shape of the second electrode, labeled Au\#2 in Figure 2, for each of the nanogaps. The photolithographically defined shape of the second electrode overlaps with the initial electrode. At this overlap the spacing between the two electrodes is controlled by the thickness of the molecular resist. A second metallic film is then deposited on the surface to form the second electrodes. The thickness of this metallic film is $50 \mathrm{~nm}$, approximately half the thickness of the metallic film used to create the initial electrodes. This ratio of initial electrode to second electrode thickness has resulted in good yields of useful devices. $(7,8)$ After removing the remaining photoresist from the second photolithography step and unwanted metal from the second evaporation step the samples are immersed in $2 \mathrm{mM}$ dodecanethiol in ethanol to form a methyl terminated self-assembled monolayer on the electrodes. Formation of this molecular film has been shown to be beneficial in passivating/stabilizing the devices. $(7,8)$ 
The samples in this work were modified with molecular resists to achieve the three different electrode-electrode spacings for study. An eicosanethiol (SC20) self-assembled monolayer molecular resist was used to create nanogaps of less than $4 \mathrm{~nm}$. This molecular resist has a thickness of $\sim 2.6 \mathrm{~nm}$ and has been used previously to create solid state nanogaps for molecular conductivity experiments. $(7,8)$ Three layers of mercaptoalkanoic acid molecules and metal ions - mercaptohexadecanoic acid/ $\mathrm{Cu}^{2+} /$ mercaptohexadecanoic acid/ $\mathrm{Cu}^{2+} /$ hexadecanethiol -were used to form a molecular resist that was nominally $6 \mathrm{~nm}$ thick. $(10,13)$ The growth of these multilevel mercaptoalkanoic acid molecular films has been extensively studied.(13) The $20 \mathrm{~nm}$ solid state nanogaps were fabricated utilizing 50-mer thiol-modified oligonucleotides as has been detailed previously.(9)

Figure 2a shows a schematic of the nanogap structure with some important features labeled. Figure $2 \mathrm{~b}$ shows a schematic emphasizing the electrode-electrode interface of the nanogap with several dimensions labeled. The blue arrow in Figure $2 b$ highlights the electrode-electrode spacing. The electrode-electrode interface can be imaged to verify the electrode-electrode spacing, at least for spacings greater than $4 \mathrm{~nm}$.(7) Figure $2 \mathrm{c}$ shows a scanning electron micrograph of a representative electrode-electrode interface fabricated with a $6 \mathrm{~nm}$ thick molecular resist. The excitation (Au\#1) and collection (Au\#2) electrodes are labeled in this figure and a blue arrow highlights the spacing between the electrodes (darker region between the electrodes in the image). Though exact measurements with SEM are difficult on this size scale the spacing was measured to be approximately $6 \pm 2 \mathrm{~nm}$. A similar procedure was used to verify the separation of solid state nanogaps fabricated with $20 \mathrm{~nm}$ think molecular resist. (9) In this case SEM imaging of the structures enabled the electrode-electrode spacing to be quantified as $20 \pm 5 \mathrm{~nm}$. No quantification of the electrode-electrode spacing for structures fabricated with molecular resists less than $4 \mathrm{~nm}$ thick was possible with SEM, though electronic measurements have verified that a spacing of a few nanometers exists.(7) Here structures fabricated with $2.6 \mathrm{~nm}$ thick molecular resist will be described as having an electrode-electrode spacing of less than $4 \mathrm{~nm}$. This is done because the exact spacing between electrodes is not known and in our hands all devices fabricated with molecular resists less than $4 \mathrm{~nm}$ thick performed poorly for electrochemical measurements as compared to those fabricated with 6 $\mathrm{nm}$ thick resists.

After fabrication the devices were sealed using a custom fabricated PDMS gaskets to create a reservoir around the electrode-electrode interfaces. The reservoirs were filled with an ionic aqueous solutions (Dulbecco's phosphate buffered saline (PBS)). A Ag/AgCl electrode was immersed in this ionic solution to act as a reference electrode. A schematic of the experimental set-up is shown in Figure $3 \mathrm{a}$. Figure $3 \mathrm{~b}$ and $3 \mathrm{c}$ are schematic representations of the volume of solution between the electrodes and the expected area of the active portion of the collection electrode, respectively, with dimensions to highlight some of the physical parameters used for quantification in this report. In these experiments, the potential on the initial or excitation electrode was swept while the current on the second or collection electrode was monitored. This is similar to the way in which electronic property measurements were taken with dry samples in previous research $(7,8)$ except here the sample was immersed in an ionic solution and a $\mathrm{Ag} / \mathrm{AgCl}$ reference electrode was included. The reference electrode reduces ionic coupling between the electrodes reducing the magnitude of the charging current as compared to experiments performed without a reference electrode.

The response of the solid state nanogaps in ionic aqueous solution differs significantly from the response of the dry structures, at least for structures with electrode-electrode spacings greater than $4 \mathrm{~nm}$. This report focuses on the variations observed with different electrodeelectrode spacing as even small, a few nanometer, change in the spacing can have significant affect on device response. Figure 4 shows the response of representative solid state nanogaps with electrode-electrode spacings of a) $<4 \mathrm{~nm}$, b) $6 \mathrm{~nm}$ and c) $20 \mathrm{~nm}$. Figure $4 \mathrm{a}$ shows the 
current-voltage traces collected for a representative solid state nanogap fabricated with an electrode-electrode spacing of less than $4 \mathrm{~nm}$. Solid state nanogaps of this size scale exhibit similar electronic behavior in ionic solution as they did when dry. The predominant mechanism of charge transfer is through tunneling-type mechanisms as seen for the dry solid state nanogaps. $(7,8)$ These tunneling-type mechanisms have a current response that is independent of the scan rate or of the addition of electroactive molecules. Specifically with these small electrode-electrode spacings very little hysteresis or splitting of the current signal due to double layer charging was observed even at high scan rates and very little charge transfer was observed that can be attributed to redox reactions. Note that the CVs in Figure 4a are for relatively fast sweep rates as compared to those shown for the 6 and $20 \mathrm{~nm}$ samples. This is done to emphasize the lack of scan rate dependence for this structure. It is expected that at this electrode-electrode spacing the dodecanethiol films, a dodecanethiol film is about $1.4 \mathrm{~nm}$ thick(14), used to stabilize the electrodes excludes most of the solution and redox active molecules from the nanogap. This exclusion from the nanogap is expected to result in the unexpected lack of electrochemical response that was observed for solid state nanogaps with less than $4 \mathrm{~nm}$ electrode-electrode spacings. The Debye layer for the PBS solution used is less than one nanometer, so even at these extremely small spacings a complete double layer should be formed, meaning that very little ionic screening was expected or observed.

Figure $4 \mathrm{~b}$ shows the $\mathrm{CV}$ trace for a representative solid state nanogap fabricated with a $6 \mathrm{~nm}$ molecular resist when immersed in a PBS solution with $0,100 \mu \mathrm{M}$ dopamine. These $\mathrm{CV}$ were collected by varying potential of the excitation electrode at a constant rate from $-0.5 \mathrm{~V}$ to +0.5 $\mathrm{V}$ compared to $\mathrm{Ag} / \mathrm{AgCl}$ and back over 2 seconds (scan rate $=1.0 \mathrm{~V} / \mathrm{s}$ ). After the $\mathrm{CV}$ was collected the potential of the excitation electrode was held at the starting potential, in this case $-0.5 \mathrm{~V}$ vs. $\mathrm{Ag} / \mathrm{AgCl}$, until an equilibrium condition was reached, in this case six seconds. Several differences are apparent between the response of the $6 \mathrm{~nm}$ device compared to the response of the $<4 \mathrm{~nm}$ nanogap. The collected current has a much smaller magnitude; the collected current is different in character; the collect current is dependent on scan rate (data not shown), and the collected current is dependent on the concentration of the redox active molecule, in this case dopamine. At molecular resist thicknesses of more than $4 \mathrm{~nm}$, no molecular conductivity or tunneling type currents are observed or expected for $\mathrm{sp}^{3}$ hybridized systems. $(7,15)$ Instead the current response in ionic solution is similar to that observed in traditional electrochemical experiments with a background current generated by the charging and discharging of the double layer and variations in surface charges at the electrode. So a portion of the current response of the $6 \mathrm{~nm}$ solid state nanogaps in ionic solution is similar to the non-Faradic or "background" charging current observed in cyclic voltammetry experiments at traditional microelectrodes. In this data set the CVs for the $6 \mathrm{~nm}$ structure in PBS before and after immersion of the device in $100 \mu \mathrm{M}$ dopamine solution are shown and are different, as expected. A "clean" device, a device before immersion in any dopamine solution, has a CV that is dominated by capacitive coupling between the electrodes (light blue trace in Figure 4b). Upon immersion in the dopamine solution, dopamine adsorbs to the device, as expected.(16) This adsorption of dopamine to the electrode surface causes a variation in the surface chemistry and this leads to a variation in the electrochemical background current observed by comparing the "before" and "after" PBS CVs for the $6 \mathrm{~nm}$ device. To verify that the variations in the observed signals are from the species in solution and not changes in the structure of the nanogap it is critical to collect before and after data for each condition. While the adsorbed species can be removed with prolonged immersion of the electrodes in PBS solution while sweeping the potential of the electrode, this treatment is not practical or necessary. Instead before data collection the solid state nanogap to be tested was immersed in an aqueous solution containing a few tens of micromolar of dopamine for at least twenty minutes. While this reduced the sensitivity of the structures to dopamine, it improved the stability of the charging current or electrochemical background current enabling reproducible cyclic voltammagrams (CV) to be 
collected in PBS solution before and after immersion in solutions of dopamine even solutions with high concentrations $(100 \mu \mathrm{M})$ of dopamine.

The maroon trace in Figure $4 \mathrm{~b}$ shows the CV response of the $6 \mathrm{~nm}$ nanogap immersed in a 100 $\mu \mathrm{M}$ aqueous dopamine solution. At this electrode-electrode spacing the addition of an electroactive species varied the response of the electrodes. Significant oxidation current was observed when the collection electrode was positive with respect to the excitation electrode and a reduction current was observed for negative potentials of the collection electrode with respect to the excitation electrode. Note that the potential of the collection electrode was held at $0 \mathrm{~V}$ with respect to $\mathrm{Ag} / \mathrm{AgCl}$ throughout the experiment. At this relatively high concentration of dopamine the electrochemical response did not show a typical diffusion limited electrochemical response but instead it continually increased with increasing potential. This devices was tested using lower concentration of dopamine, Figure 5, and at these lower concentration had a CV response more similar to what is expected for dopamine.(16) Note the two features around zero volts in Figure 4b. In the typical experiment the electrodes are held at a constant potential for six seconds between the collection of CVs. By eliminating the resting time between CVs and increasing the scan rate the magnitude of this peak can be reduced. For this reason these features are currently being attributed to desorption of dopamine from the electrode surface. To ensure that the observed signal was from electrochemical reaction, this same solid state nanogap was immersed in a solution of phenylalanine, a molecule of similar size and character to dopamine, but that is not electroactive. No variation in response, as compared to PBS, was observed for phenylalanine concentration up to $1 \mathrm{mM}$, the largest concentration tested (data not shown). This reinforced the concept that the collected signal was due to electrochemistry and not a molecular conductivity mechanism.

Figure $4 \mathrm{c}$ shows $\mathrm{CV}$ traces from a representative nanogap sensor fabricated with a $20 \mathrm{~nm}$ thick molecular resist upon immersion in a PBS solution containing $0,100 \mu \mathrm{M}$ dopamine. These traces were generated with the same condition used in Figure $4 \mathrm{~b}$, by sweeping the potential of the excitation electrode and recording at the collection electrode with a scan rate of $1.0 \mathrm{~V} / \mathrm{s}$. A background signal was observed for this structure, but at this separation, $20 \mathrm{~nm}$, and these conditions very little signal was collected that can be attributed to an electrochemical reactions, even with a $100 \mu \mathrm{M}$ dopamine solution. Note that steady state electrochemical measurements could be made with this $20 \mathrm{~nm}$ device in the $100 \mu \mathrm{M}$ dopamine solution. This measurement resulted in $10 \mathrm{pA}$ of current being collected for the $20 \mathrm{~nm}$ device, similar measurements for the $6 \mathrm{~nm}$ device in a $100 \mu \mathrm{M}$ dopamine solution resulted in $120 \mathrm{pA}$ of current being collected. Steady state measurements will be further discussed below. For completeness an example CV from a microelectrode immersed in a $100 \mu \mathrm{M}$ dopamine solution is included, Figure $4 \mathrm{~d}$.

From Figure 4 it is apparent that the solid state nanogaps with $6 \mathrm{~nm}$ electrode-electrode spacings have significantly more electrochemical activity than the other spacings of solid state nanogaps tested. Since excluding the redox active species from the $<4 \mathrm{~nm}$ nanogap eliminates most of the electrochemical activity and since increasing to $20 \mathrm{~nm}$ electrode-electrode spacing also eliminates most of the electrochemical activity it is expect that most of the observed electrochemical activity is from the nanogap itself and little is due to the other portions of the electrodes exposed to solution. From this data it is expected that mass transfer restriction at the $6 \mathrm{~nm}$ nanogap leads to significant cycling of the redox active species inside the nanogap.

To further examine this variation due to the addition of a redox active molecule the direction of the potential sweep was reversed and the concentration of dopamine reduced. The direction of the potential sweep was reversed because holding the excitation electrode at $+0.5 \mathrm{~V}$ with respect to the $\mathrm{Ag} / \mathrm{AgCl}$ electrode changed the local chemical environment by building a concentration of dopamine-o-quinone in close proximity to the electrodes. Switching the direction of the potential sweep eliminated this issue and gave more interruptible information 
for smaller concentration of dopamine. Figure 5a shows CVs from a representative $6 \mathrm{~nm}$ solid state nanogap for solutions of $0,2,25 \mu \mathrm{M}$ dopamine. A concentration dependent response was observed. To improve understanding of this data the response due to PBS is subtracted from the response to the $2 \mu \mathrm{M}$ dopamine solution and the potential of collection electrode is plotted with respect to the excitation electrode resulting in the trace in Figure 5b. The blue arrow highlights an oxidation peak and the red arrow a reduction peak for the dopamine. The positions for the oxidation and reduction peaks of dopamine fall within the range found in the literature for Au electrodes.(17-26) The magnitude of the electrochemical response can be used to further evaluate the magnitude of the "enhanced" electrochemical response.

The electrochemical data collected at these nanogap structures is not well modeled by traditional electrochemical models. To emphasize that the collected current does not follow commonly accepted electrochemical trends, the relationship between electrode area and the collected peak current was investigated. If it is assumed that the response follows RandlesSevcik behavior (diffusion limited transfer of the electroactive species) and that the active area is only the portion of the collection electrode that comprises the nanogap (see Figure 3c). This enables the Randles-Sevcik equation (1) to be used to approximate the expected peak current of the electrode given a set of conditions.

$$
i_{p}=2.69 \times 10^{5} * n^{3 / 2} * v^{1 / 2} * D_{0}{ }^{1 / 2} * C_{0} * A_{e}
$$

Where $A_{e}$ is the effective electroactive area, $i_{p}$ is the expected peak current, $n$ is the number of electrons transferred in the electrochemical reaction ( 2 for dopamine), $D_{0}$ is the diffusion constant of the electroactive species (for dopamine $7.6 \times 10^{-10} \mathrm{~m}^{2} / \mathrm{s}$ was used(27)), $v$ is the rate of the potential sweep $(1 \mathrm{~V} / \mathrm{s})$ and $C_{0}$ is the concentration of the electroactive species. Calculating the expected peak current for the portion of the collection electrode at the nanogap $\left(A_{e}=1 \times 10^{-13} \mathrm{~m}^{2}\right.$, Figure $\left.3 \mathrm{c}\right)$ with these conditions for $2 \mu \mathrm{M}\left(\right.$ or $\left.2 \times 10^{-3} \mathrm{moles} / \mathrm{m}^{3}\right)$ dopamine would result in an expected peak current of $\sim 4 \times 10^{-15} \mathrm{~A}$. This is significantly smaller than the measured peak current of $1.5 \times 10^{-11} \mathrm{~A}$ (Figure $5 \mathrm{~b}$ ). Even if all of the collection electrode area exposed to solution, though it is expected only portion at the nanogap contributes to the electrochemical activity based on the results from the $20 \mathrm{~nm}$ structures, the measured current still exceeds the expected current based on the Randles-Sevcik model by an order of magnitude. It is obvious from this simple analysis that traditional electrochemical models do not explain the results from these solid state nanogaps. Note that the Randles-Sevcik equation is for diffusion-limited signals, but similarly poor results are obtained for traditional electrochemical models based on adsorption of the electroactive species.

In previous research by Lemay and co-workers, it was proposed that steady state current due to the cycling of electrochemically active species at electrodes spaced by a few microns to a few nanometers in a closed system could be estimated by the following equation(6):

$$
i_{c y c l}=\frac{<n>^{*} D^{*} z e}{d^{2}}
$$

Where $i_{\text {cycl }}$ is the steady state redox current collected, $\langle n\rangle$ is the number of molecules in solution between the electrodes, $D$ is the diffusion coefficient of dopamine $\left(D=7.6 \times 10^{-10}\right.$ $\mathrm{m}^{2} / \mathrm{s}$ was used(27)), $z e$ is the charge transfer per redox reaction $\left(z e=3.2 \times 10^{-19} \mathrm{C}\right)$, and $d$ is the distance between the electrodes $\left(d=6 \times 10^{-9} \mathrm{~m}\right)$. In the situation with $2 \mu \mathrm{M}$ of dopamine and the fabricated systems, $\langle n\rangle$, the number of molecules between the electrodes would be equal to $\mathbf{\sim 0 . 7 4}$ molecules. The volume of solution between electrodes is estimated to be $6 \times$ $10^{-22} \mathrm{~m}^{3}$ or $6 \times 10^{-19} \mathrm{~L}$ (Figure $3 \mathrm{c}$ ). By calculating the expected steady state current using 
these values results in an $i_{c y c l}$ of $5 \mathrm{pA}$. For comparison the steady state current for the $6 \mathrm{~nm}$ solid state nanogap was collected by holding the potential of the collection electrode at a constant $+0.5 \mathrm{~V}$ with respect to the excitation electrode. This resulted in a $10 \mathrm{pA}$ steady state current being collected for the $6 \mathrm{~nm}$ solid state nanogaps when immersed in the $2 \mu \mathrm{M}$ dopamine solution. This is in good agreement with the calculated value especially considering that the equation (2) was developed for a closed system where the molecules are forced to diffuse between the two electrodes. Note that the steady state measurement for the $20 \mathrm{~nm}$ device was $10 \mathrm{pA}$ and for the $6 \mathrm{~nm}$ device was $120 \mathrm{pA}$ in a $100 \mu \mathrm{M}$ dopamine solution (from above). From equation 2, the $6 \mathrm{~nm}$ device would be expected to have 11 times the steady state response of the $20 \mathrm{~nm}$ device, so these measurements are in good agreement with this model. Note that while this model is expected to oversimplify much of the physics at the electrode surface, it gives good results especially compared to the traditional electrochemical models.

This paper details initial experiments developing planar solid state nanogaps for electrochemical detection. The initial experiments show a large "electrochemical enhancement factor" for solid state nanogaps with electrode-electrode spacings of $6 \mathrm{~nm}$. This enhanced signal is due to cycling of the redox active molecules at the confined geometry of the nanogap and enables monitoring of just a few molecules. This study also highlights a limit to scaling for these devices, at least under these conditions. When devices are scaled to be only a few nanometers, properties that are typically trivial such as molecule size become important and can lead to significant variation in performance. While the initial studies have shown an "electrochemical enhancement factor" and are promising, substantial research still needs to be performed to optimize and then validate these techniques for monitoring electrochemical species in more complex chemical environments.

\section{Acknowledgments}

The authors would like to thank Prof. R.M. Wightman and Prof. L.A. Sombers for helpful discussion. G.S.M, M.K.Z. and B.P.M. would like to thank NIH (DA023586) for funding. Micro- and nanofabrication was preformed in the Penn State Nanofabrication Facility, the Biomedical Microsensors Laboratory at NCSU and the North Carolina State Nanofabrication Facility. The Penn State Nanofabrication Facility is a member of the National Science Foundation sponsored National Nanofabrication Infrastructure Network.

\section{References}

1. Fan FRF, Bard AJ. Science 1995 Feb 10;267:871. [PubMed: 17813918]

2. Fan FRF, Kwak J, Bard AJ. Journal of the American Chemical Society 1996 Oct 9;118:9669.

3. Niwa O, Morita M, Tabei H. Electroanalysis 1991 Apr;3:163.

4. Goluch ED, Wolfrum B, Singh PS, Zevenbergen MAG, Lemay SG. Analytical and Bioanalytical Chemistry 2009;394:447. [PubMed: 19125239]

5. Dam VAT, Olthuis W, van den Berg A. Analyst 2007;132:365. [PubMed: 17554417]

6. Wolfrum B, Zevenbergen M, Lernay S. Analytical Chemistry 2008 Feb 15;80:972. [PubMed: 18193890]

7. McCarty GS. Nano Letters 2004 Aug;4:1391.

8. McCarty GS. Journal Of Applied Physics 2006 Mar 15;99

9. Moody B, McCarty GS. Applied Physics Letters 2009;94:122104.

10. Hatzor A, Weiss PS. Science 2001 Feb 9;291:1019. [PubMed: 11161210]

11. Anderson ME, et al. Advanced Materials 2006 Apr 18;18:1020.

12. Subramanian S, McCarty GS, Catchmark JM. Journal of Microlithography Microfabrication and Microsystems 2005 Oct-Dec; 4

13. Daniel TA, Uppili S, McCarty G, Allara DL. Langmuir 2007 Jan 16;23:638. [PubMed: 17209615]

14. Bumm LA, Arnold JJ, Dunbar TD, Allara DL, Weiss PS. Journal of Physical Chemistry B 1999 Sep 23;103:8122. 
15. Rampi MA, Whitesides GM. Chemical Physics 2002 Aug 1;281:373.

16. Zachek MK, Hermans A, Wightman RM, McCarty GS. Journal of Electroanalytical Chemistry 2008;614:113. [PubMed: 19319208]

17. Heien M, Johnson MA, Wightman RM. Analytical Chemistry 2004 Oct 1;76:5697. [PubMed: 15456288]

18. Heien M, et al. Proceedings of the National Academy of Sciences of the United States of America 2005 Jul 19;102:10023. [PubMed: 16006505]

19. Sombers LA, Colliver TL, Ewing AG. Chromaffin Cell: Trnsmitter Biosynthesis, Storage, Release, Actions, and Informatics 2002; vol. 971:86-88.

20. Sombers LA, et al. Journal of Neuroscience 2004 Jan 14;24:303. [PubMed: 14724228]

21. Liu T, Li MX, Li QY. Talanta 2004 Jul 8;63:1053. [PubMed: 18969533]

22. Venton BJ, Michael DJ, Wightman RM. Journal of Neurochemistry 2003 Jan;84:373. [PubMed: 12558999]

23. Runnels PL, Joseph JD, Logman MJ, Wightman RM. Analytical Chemistry 1999 Jul 15;71:2782. [PubMed: 10424168]

24. Cahill PS, et al. Analytical Chemistry 1996 Sep 15;68:3180. [PubMed: 8797378]

25. Kawagoe KT, Garris PA, Wightman RM. Journal of Electroanalytical Chemistry 1993 Nov 15;359:193.

26. Pihel K, Walker QD, Wightman RM. Analytical Chemistry 1996 Jul 1;68:2084. [PubMed: 9027223 ]

27. Cragg SJ, Rice ME. Trends in Neurosciences 2004;27:270. [PubMed: 15111009] 


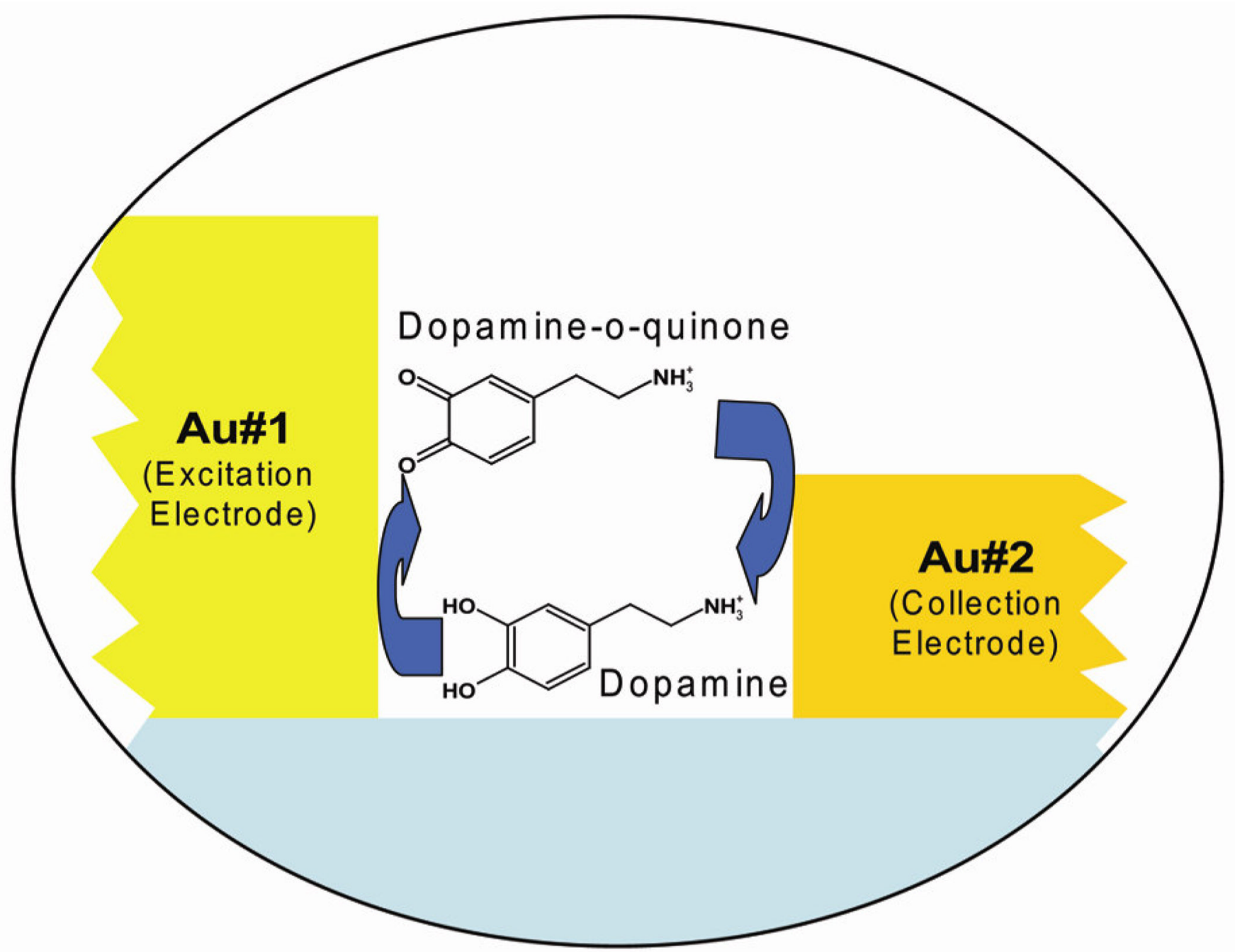

Figure 1.

A schematic representation of electrochemical cycling of dopamine in a nanogap. The dopamine molecule is oxidized to dopamine ortho-quinone at one electrode and then reduced back to dopamine at the other electrode. This cycling can happen repeatedly for a single molecule. 


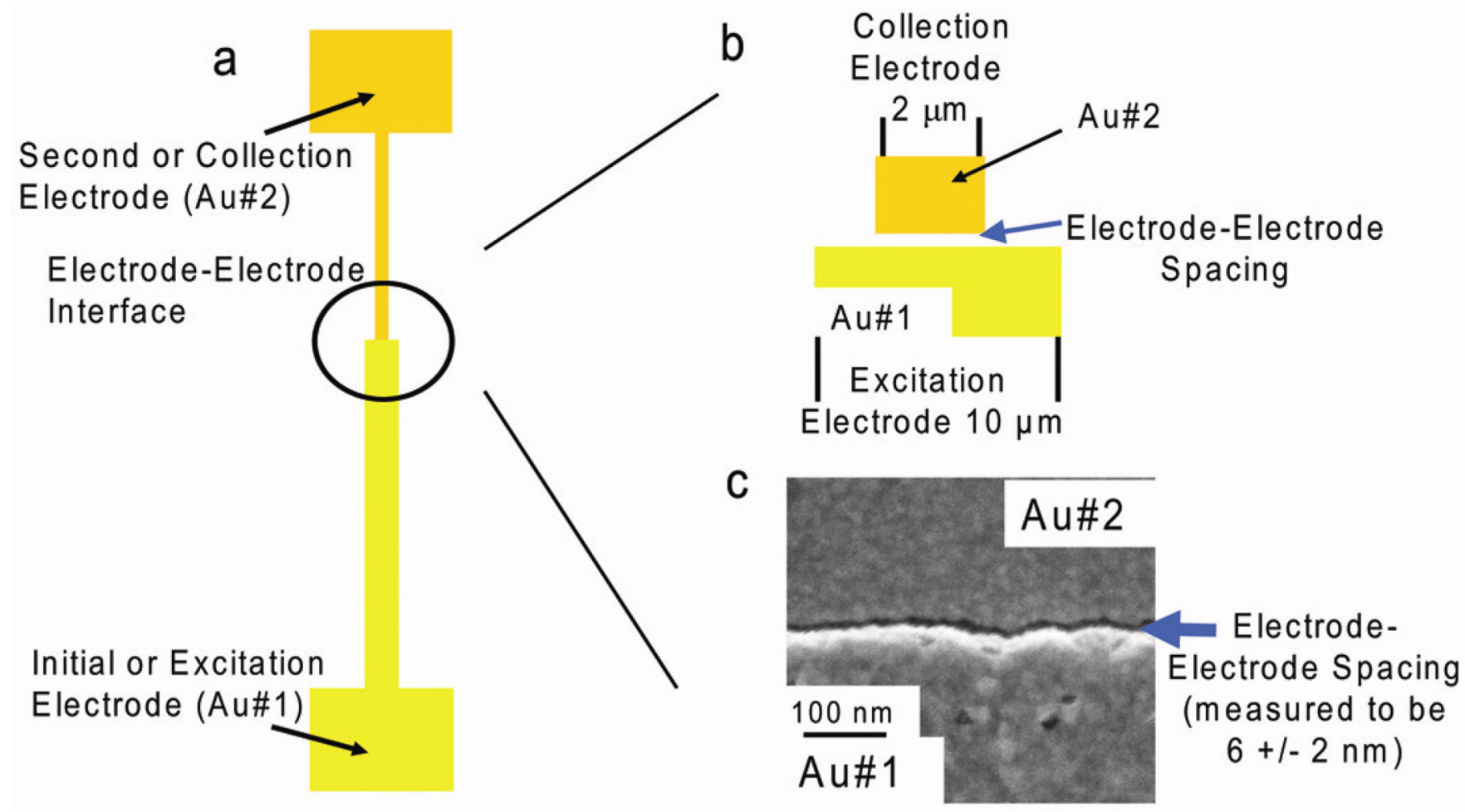

Figure 2.

A pictorial overview of the planar solid state nanogap structures used. a) A schematic overview of the planar solid state nanogap with some important features labeled. b) A schematic showing the electrode-electrode interface portion of the solid state nanogap with important dimensions enumerated. The dimensions of the schematic are not too scale. c) Electron microgaph of a portion of the electrode-electrode interface for a solid state nanogap fabricated with a $6 \mathrm{~nm}$ thick molecular resist. The spacing was measured to be $\sim 6 \pm 2 \mathrm{~nm}$. 


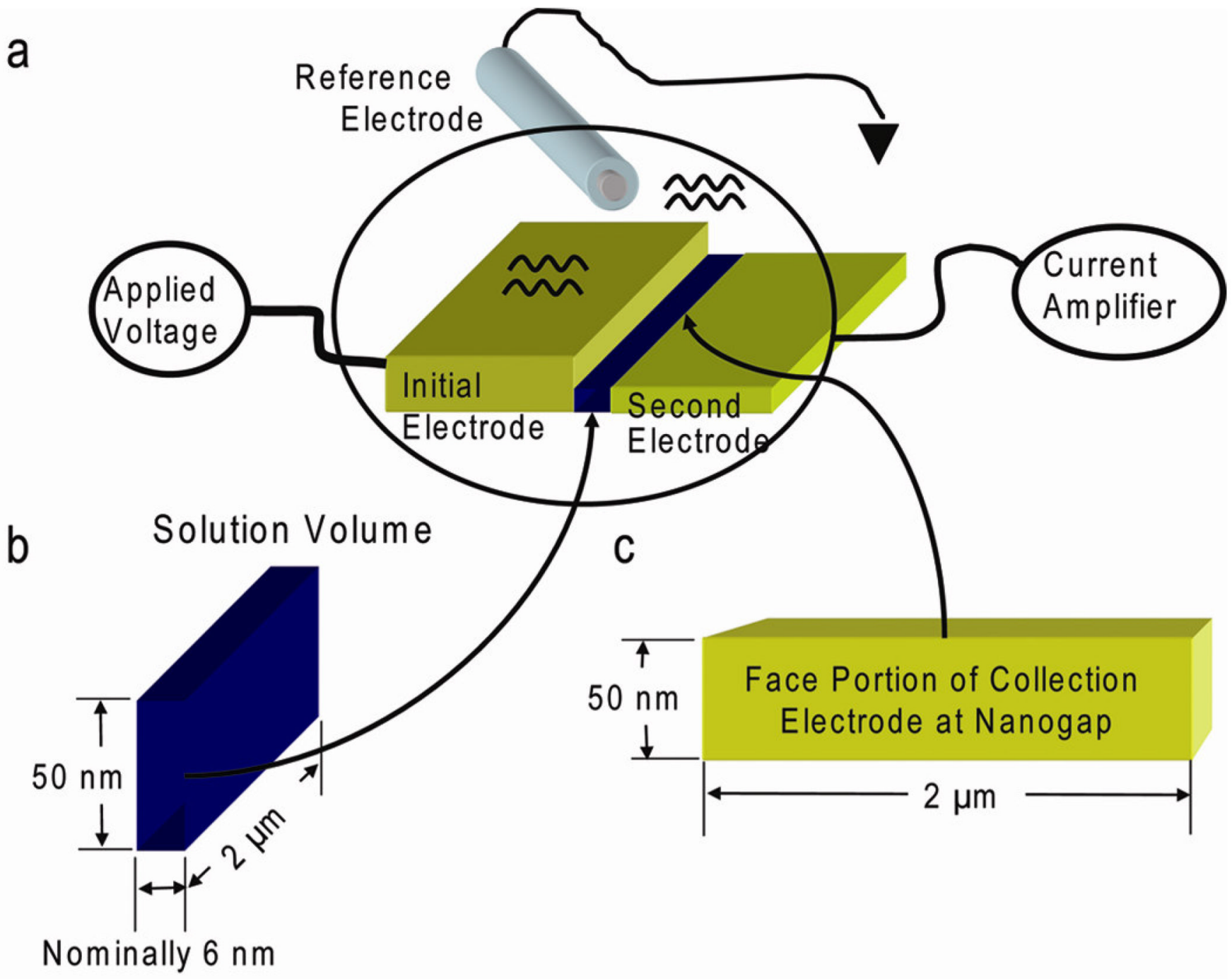

Figure 3.

a) A schematic of the experimental set-up used. The electrode-electrode interface is exaggerated in this schematic. The b) volume of solution between the electrodes at the electrode-electrode interface and c) the area of the face of the collection electrode that comprises the solid state nanogap are extracted and dimensions add for emphasis. 
a

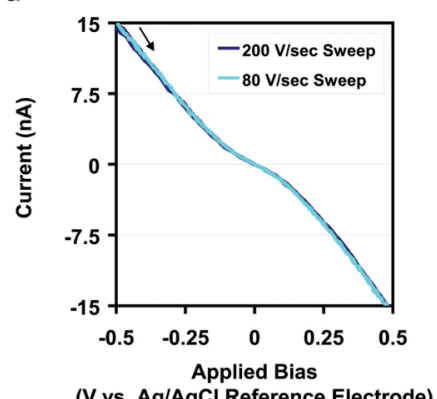

b

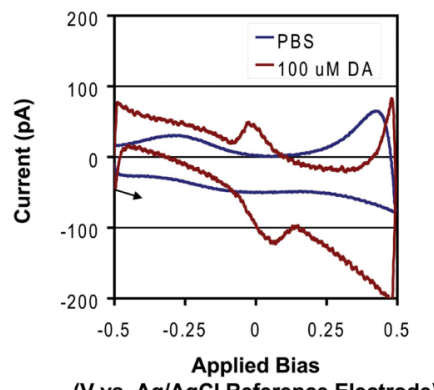

(V vs. Ag/AgCl Reference Electrode)
C

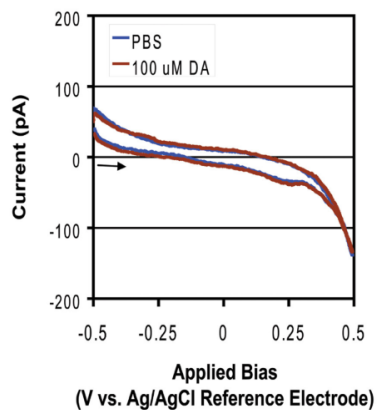

d

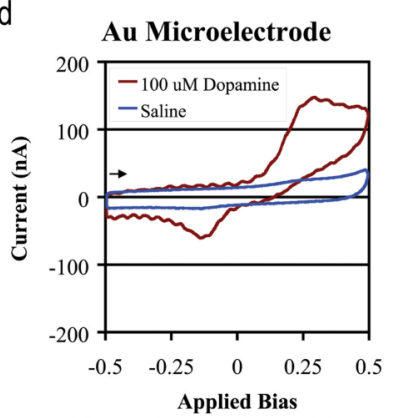

(V vs. Ag/AgCl Reference Electrode)

Figure 4.

The current-voltage responses in ionic solutions of solid state nanogaps with a) $<4 \mathrm{~nm}, \mathrm{~b}$ ) nominally $6 \mathrm{~nm}$ and c) nominally $20 \mathrm{~nm}$ electrode-electrode spacings. These data were collected by scanning the potential of the excitation electrode a) $80,200 \mathrm{~V} / \mathrm{s}$ and b),c) $1 \mathrm{~V} / \mathrm{s}$ while monitoring current at the collection electrode. d) An example CV from a Au microelectrode immersed in a $100 \mu \mathrm{M}$ dopamine aqueous solution collected by sweeping the working electrode from $-0.5 \mathrm{~V}$ to $+0.5 \mathrm{~V}$ and back at $1 \mathrm{~V} / \mathrm{s}$. 

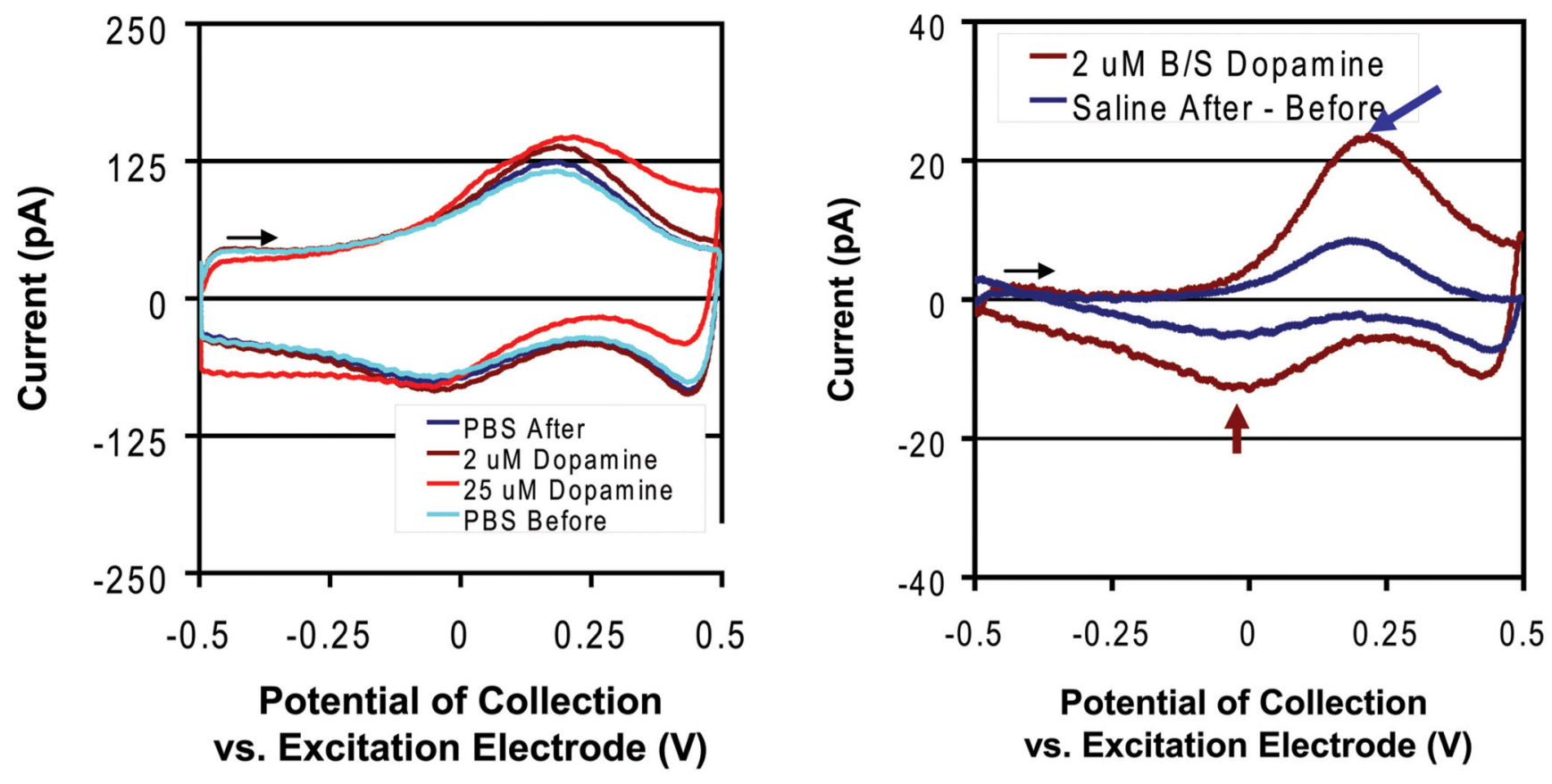

Figure 5.

a) The current-voltage response of a representative nominally $6 \mathrm{~nm}$ solid state nanogap in ionic solution with 0,2 , and $25 \mu \mathrm{M}$ dopamine. These data were collected by sweeping the potential of the collection electrode at $1 \mathrm{~V} / \mathrm{s}$ while monitoring current at the collection electrode. b) Background subtracted current-voltage responses, $2 \mu \mathrm{M}$ dopamine - before PBS response and the after PBS - before PBS response from part (a), for the representative solid state nanogap. 